\title{
Dispersion representation of the D-term form factor in deeply vir- tual Compton scattering
}

\author{
B. Pasquini ${ }^{1, a}$ \\ ${ }^{1}$ Dipartimento di Fisica, Università degli Studi di Pavia, Pavia, Italy, \\ and Istituto Nazionale di Fisica Nucleare, Sezione di Pavia, Pavia, Italy
}

\begin{abstract}
We review the dispersion relation formalism for the deeply virtual Compton scattering amplitudes, and derive a dispersive representation of the D-term form factor, using unsubtracted $t$-channel dispersion relations. Results for the D-term form factor as function of $t$ as well as at $t=0$ are shown in comparison with available model predictions and phenomenological parametrizations.
\end{abstract}

\section{Introduction}

Dispersion relations (DRs) provide an useful framework to connect different observables and to extract nucleon structure quantities. In particular, DRs for Compton scattering processes, with both real and virtual photons, had wide applications for the prediction and extraction from experimental data of lowenergy properties of hadron systems [1-5]. More recently, the dispersion formalism has been extended to describe the virtual Compton scattering process in the deep inelastic region [6-12]. In particular, it was shown that the amplitudes for deeply virtual Compton scattering (DVCS) satisfy subtracted DRs at fixed $t$ with the subtraction function defined by the D-term form factor [6-8]. The D term was originally introduced to complete the parametrization of the generalized parton distributions (GPDs) in hard exclusive reactions in terms of double distributions, and restore the polynomiality property of the singlet moments of unpolarized GPDs [13]. This term turned out to be a crucial contribution in the phenomenological description of DVCS observables, where different forms have been assumed with parameters tuned to DVCS data $[9,14]$.

Here we review the dispersive representation of the D-term form factor in terms of DRs in the $t$ channel, as recently proposed in Ref. [15]. The advantage of this dispersive representation is to provide a microscopic interpretation of the physical content of the D-term form factor in terms of $t$-channel exchanges with the appropriate quantum numbers.

In Section 2, we review the derivation of the $s$-channel subtracted dispersion relations for the DVCS amplitudes. In Section 3, we derive $t$-channel DRs for the D-term form factor, and give the ingredients for the explicit calculation, by saturating the unitarity relation for the $t$-channel amplitudes with twopion intermediate states. We then discuss the dispersive predictions for the D-term form factor in Section 4 , and we conclude summarizing our results.

ae-mail: barbara.pasquini@unipv.it 


\section{Subtracted dispersion relations in the $s$-channel}

We consider the DVCS process

$$
\gamma^{*}(q) N(p) \rightarrow \gamma\left(q^{\prime}\right) N\left(p^{\prime}\right),
$$

where the variables in brackets denote the four-momenta of the participating particles. The familiar Mandelstam variables are $s=(p+q)^{2}, t=\left(q-q^{\prime}\right)^{2}, u=\left(q-p^{\prime}\right)^{2}$, and are constrained by $s+u+t=$ $2 M_{N}^{2}-Q^{2}$, with $M_{N}$ the nucleon mass and $Q^{2}=-q^{2}$. We will consider the Bjorken regime, where the photon virtuality $Q^{2}$ and $s$ are large, and $-t \ll s, Q^{2}$.

The DVCS amplitude reads

$$
T_{\lambda_{\gamma}^{\prime} \lambda_{N}^{\prime}, \lambda_{\gamma} \lambda_{N}}=\varepsilon_{\mu}\left(q, \lambda_{\gamma}\right) \varepsilon_{\nu}^{*}\left(q^{\prime}, \lambda_{\gamma}^{\prime}\right) H_{\lambda_{N}^{\prime}, \lambda_{N}}^{\mu \nu},
$$

where $\varepsilon_{\lambda_{\gamma}}\left(\varepsilon_{\lambda_{\gamma}}^{*}\right)$ is the polarization vector of the incoming virtual (outgoing real) photon and the DVCS tensor is defined as the nucleon matrix element of the $T$-product of two electromagnetic currents:

$$
H_{\lambda_{N}^{\prime}, \lambda_{N}}^{\mu \nu}=-i \int \mathrm{d}^{4} x e^{-i(q \cdot x)}\left\langle N\left(p^{\prime}, \lambda_{N}^{\prime}\right)\right| T\left[J^{\mu}(x) J^{\nu}(0)\left|N\left(p, \lambda_{N}\right)\right\rangle,\right.
$$

where $\lambda_{N}\left(\lambda_{N}^{\prime}\right)$ is the helicity of the incoming (outgoing) nucleon.

The DVCS amplitude for unpolarized nucleon and at leading order in $Q$ can be parametrized as

$$
\begin{aligned}
T_{\lambda_{\gamma}^{\prime} \lambda_{N}^{\prime}, \lambda_{\gamma} \lambda_{N}} & =\varepsilon_{\mu}\left(q, \lambda_{\gamma}\right) \varepsilon_{v}^{*}\left(q^{\prime}, \lambda_{\gamma}^{\prime}\right) \frac{\left(-g_{\perp}^{\mu \nu}\right)}{2} \\
& \times\left[\bar{u}\left(p^{\prime}, \lambda_{N}^{\prime}\right) \gamma \cdot n u\left(p, \lambda_{N}\right) \sum_{q} e_{q}^{2} C^{q}-\bar{u}\left(p^{\prime}, \lambda_{N}^{\prime}\right) u\left(p, \lambda_{N}\right) \frac{1}{M_{N}} \sum_{q} e_{q}^{2} F^{q}\right],
\end{aligned}
$$

where we introduced the lightlike vector $n^{\mu}=1 /\left(\sqrt{2} P^{+}\right)(1,0,0,-1)$, with $P=\left(p+p^{\prime}\right) / 2$, and the symmetric tensor $g_{\perp}^{\mu \nu}=g^{\mu \nu}-n^{\mu} \tilde{p}^{v}-n^{v} \tilde{p}^{\mu}$, with $\tilde{p}^{\mu}=P^{+} / \sqrt{2}(1,0,0,1)$. Furthermore, the light-front component for a generic four-vector $a^{\mu}$ is defined as $\left(a^{0}+a^{3}\right) / \sqrt{2}$. In Eq. (4), the invariant amplitudes $C^{q}$ and $F^{q}$ are given by

$$
C^{q}(\xi, t)=\int_{-1}^{1} \mathrm{~d} x \frac{H^{(+)}(x, \xi, t)+E^{(+)}(x, \xi, t)}{x-\xi+i \epsilon}, \quad F^{q}(\xi, t)=\int_{-1}^{1} \mathrm{~d} x \frac{E^{(+)}(x, \xi, t)}{x-\xi+i \epsilon},
$$

with the skewedness variable defined as $\xi=Q^{2} /\left(2 s+Q^{2}\right) . H^{(+)}(x, \xi, t)=H^{q}(x, \xi, t)-H^{q}(-x, \xi, t)$ denotes the singlet $(C=+1)$ combination of nucleon helicity-conserving GPDs, and analogously for the nucleon helicity-flip GPD $E^{(+)}$. The invariant amplitudes and the GPDs in Eq. (5) depend also on the renormalization scale $\mu^{2}$, which is not explicitly displayed, and it is identified with the hard scale of the process $Q^{2}$.

In the following we will consider the invariant amplitude $F^{q}$ in the $v-t$ plane at fixed $Q^{2}$, with $v=(s-u) / 4 M_{N}=Q^{2} / 4 M_{N} \xi$. In this plane, $F^{q}$ satisfies the following fixed- $t$ subtracted relation $[6,11]$

$$
F^{q}(v, t)=F^{q}(0, t)+\frac{v^{2}}{\pi} \int_{v_{0}}^{\infty} \frac{\mathrm{d} v^{\prime 2}}{v^{\prime 2}} \frac{\operatorname{Im} F^{q}\left(v^{\prime}, t\right)}{v^{\prime 2}-v^{2}},
$$

where the lower limit of integration is $v_{0}=Q^{2} / 4 M_{N}$ and the nucleon pole term residing in this point may be considered separately. Following Refs. $[6,8]$, the subtraction function $F^{q}(0, t)$ can be related to the D-term form factor $D^{q}(t)[13]$ as follows

$$
F^{q}(0, t)=2 \int_{-1}^{+1} \mathrm{~d} z \frac{D^{q}(z, t)}{1-z}=4 D^{q}(t) .
$$




\section{$3 t$-channel dispersion relations for the D-term form factor}

The dispersive representation for the D-term form factor $D^{q}(t)$ of Eq. (7) is obtained by applying unsubtracted DRs, this time in the variable $t$ :

$$
F^{q}(0, t)=\frac{1}{\pi} \int_{4 m_{\pi}^{2}}^{+\infty} \mathrm{d} t^{\prime} \frac{\operatorname{Im}_{t} F^{q}\left(0, t^{\prime}\right)}{t^{\prime}-t}+\frac{1}{\pi} \int_{-\infty}^{-a} \mathrm{~d} t^{\prime} \frac{\operatorname{Im}_{t} F^{q}\left(0, t^{\prime}\right)}{t^{\prime}-t} .
$$

The imaginary part in the integral from $4 m_{\pi}^{2} \rightarrow+\infty$ in Eq. (8) is saturated by the possible intermediate states for the $t$-channel process, which lead to cuts along the positive- $t$ axis. For low values of $t$, the $t$-channel discontinuity is dominated by $\pi \pi$ intermediate states.

The second integral in Eq. (8) extends from $-\infty$ to $-a=-2\left(m_{\pi}^{2}+2 M_{N} m_{\pi}\right)-Q^{2}$. As we are interested in evaluating Eq. (8) for large $Q^{2}$ values and small (negative) values of $t(|t| \ll a)$, the integral from $-\infty \rightarrow-a$ is suppressed, and will be neglected in this work. Consequently, we shall saturate the integral in Eq. (8) by the contribution of $\pi \pi$ intermediate states, which turns out to be a good approximation for small $t$.

We start by decomposing the $t$-channel helicity amplitude for $\gamma^{*} \gamma \rightarrow \bar{N} N$ into a partial wave series,

$$
T_{\lambda_{\bar{N}} \lambda_{N}, \lambda_{\gamma} \lambda_{\gamma}^{\prime}}^{t}(v, t)=\sum_{J} \frac{2 J+1}{2} T_{\lambda_{N} \lambda_{\bar{N}}, \lambda_{\gamma}^{\prime} \lambda_{\gamma}}^{J\left(\gamma^{*}\right)}(t) d_{\Lambda_{N} \Lambda_{\gamma}}^{J}\left(\theta_{t}\right)
$$

where $\Lambda_{\gamma}=\lambda_{\gamma}^{\prime}-\lambda_{\gamma}, \Lambda_{N}=\lambda_{N}-\lambda_{\bar{N}}$, and $d_{\Lambda_{N} \Lambda_{\gamma}}^{J}$ are Wigner $d$-functions. As described in Ref. [15], the unitarity relation for the the partial-wave amplitudes in the $t$-channel, with only the $\pi \pi$ intermediate states, reads

$$
2 \operatorname{Im} T_{\lambda_{\bar{N}} \lambda_{N}, \lambda_{\gamma} \lambda_{\gamma}^{\prime}}^{J\left(\gamma^{*}\right)}(t)=\frac{1}{(8 \pi)} \frac{p_{\pi}}{\sqrt{t}}\left[T_{\Lambda_{\gamma}}^{J\left(\gamma^{*} \gamma \rightarrow \pi \pi\right)}(t)\right]\left[T_{\Lambda_{N}}^{J(\pi \pi \rightarrow N \bar{N})}(t)\right]^{*},
$$

where $T^{J\left(\gamma^{*} \gamma \rightarrow \pi \pi\right)}$ and $T^{J(\pi \pi \rightarrow N \bar{N})}$ are the partial-wave amplitudes for the $\gamma^{*} \gamma \rightarrow \pi \pi$ and the $\pi \pi \rightarrow N \bar{N}$ subprocesses, respectively.

The partial-wave amplitudes $T_{\Lambda_{N}=0}^{J(\pi \pi \rightarrow N \bar{N})}$ are related to the amplitudes $f_{+}^{J}(t)$ of Frazer and Fulco [16] by the relation

$$
T_{\Lambda_{N}=0}^{J(\pi \pi \rightarrow N \bar{N})}(t)=\frac{16 \pi}{p_{t}}\left(p_{t} p_{\pi}\right)^{J} f_{+}^{J}(t)
$$

The reaction $\gamma^{*} \gamma \rightarrow \pi \pi$ at large $Q^{2}$ and small $t$ can be described in a factorized form [17, 18], as the convolution of a short-distance contribution, $\gamma^{*} \gamma \rightarrow q \bar{q}$, perturbatively calculable, and nonperturbative matrix elements describing the exclusive fragmentation of a $q \bar{q}$ pair into two pions. These nonperturbative functions correspond to two-pion generalized distribution amplitudes (GDAs). In particular, one finds

$$
T_{\Lambda_{\gamma}=0}^{J\left(\gamma^{*} \gamma \rightarrow \pi \pi\right)}(t)=\sum_{q} e_{q}^{2} T_{\Lambda_{\gamma}=0}^{J\left(\gamma^{*} \gamma \rightarrow q \bar{q}\right)}(t)
$$

with

$$
T_{\Lambda_{\gamma}=0}^{J\left(\gamma^{*} \gamma \rightarrow q \bar{q}\right)}(t)=\frac{6}{2 J+1} \sum_{\substack{n=\max (1, J-1) \\ \text { odd }}} \int_{0}^{1} \mathrm{~d} z(2 z-1) \tilde{B}_{n J}(t) C_{n}^{(3 / 2)}(2 z-1),
$$

where $C_{n}^{(3 / 2)}$ are Gegenbauer polynomials, and $\tilde{B}_{n l}^{q}$ are expansion coefficients of the GDA amplitude [18-20]. Inserting the partial wave expansion of Eqs. (11) and (12) into Eq. (10), we can finally 
express the $2 \pi t$-channel contribution to $\operatorname{Im}_{t} F^{q}(v=0, t)$ as

$$
\operatorname{Im}_{t} F^{q(\pi \pi)}=-\frac{M_{N} p_{\pi}}{\sqrt{t} p_{t}^{2}} \sum_{\substack{J \\ \text { even }}} \frac{2 J+1}{2}(-1)^{J / 2} \frac{(J-1) ! !}{J ! !}\left(p_{t} p_{\pi}\right)^{J} T_{\Lambda_{\gamma}=0}^{J\left(\gamma^{*} \gamma \rightarrow q \bar{q}\right)} f_{+}^{J *}(t) .
$$

For the numerical estimate, we restrict ourselves to the $S$ - and $D$-wave contributions in Eq. (14). The partial-wave amplitudes of the $\pi \pi \rightarrow N \bar{N}$ subprocess are taken from [21]. The two-pion GDAs are calculated through DRs using the Omnès representation [19, 20, 22-24]. The results for the $S$ - and $D$-wave coefficients, for $n=1$ in the Gegenbauer expansion of (13), read

$$
\tilde{B}_{10}(t)=-B_{12}(0) \frac{3 C-\beta^{2}}{2} f_{0}(t), \quad \tilde{B}_{12}(t)=\beta^{2} B_{12}(0) f_{2}(t),
$$

where the Omnès functions $f_{0,2}$ can be related to $\pi \pi$ phase-shifts $\delta_{0,2}^{0}(t)$ using Watson theorem and dispersion relations [19]. In Eq. (15), the constant $C$ is taken from Ref. [20], using the estimate from the instanton model [25] at low energies, $C=1+b m_{\pi}^{2}+O\left(m_{\pi}^{4}\right)$ with $b \approx-1.7 \mathrm{GeV}^{-2}$, while the coefficient $B_{12}(0)$ is obtained using the crossing relations between the quark $2 \pi$ DA's and the corresponding parton distributions in the pion, i.e.

$$
B_{12}(0)=\frac{10}{9} \int \mathrm{d} x \times \frac{1}{N_{f}} \sum_{f}\left[q_{\pi}^{f}(x)+\bar{q}_{\pi}^{f}(x)\right] .
$$

As final result, taking into account only the contribution with $J=0$ and $J=2$, Eq. (14) simplifies to

$$
\operatorname{Im}_{t} F^{q(\pi \pi)}=\frac{3 M_{N} p_{\pi}}{2 \sqrt{t} p_{t}^{2}} B_{12}(0)\left[\left(3 C-\beta^{2}\right) f_{0}(t) f_{+}^{0 *}(t)+\left(p_{\pi} p_{t}\right)^{2} \beta^{2} f_{2}(t) f_{+}^{2 *}(t)\right] .
$$

In Eq. (17), the dependence on the renormalization scale enters only through the coefficient $B_{12}^{q}$ evaluated at $t=0$, and therefore is disjoined from the $t$ dependence of the amplitude. Furthermore, the coefficients $B_{12}^{q}$ evolve in the same way as the quark momentum fraction in the pion, in accordance with Eq. (16).

We note that the restriction to $n=1$ in the Gegenbauer expansion of the GDAs corresponds to a similar restriction for the D-term form factor. As a matter of fact, the D-term $D(z, t)$ in Eq. (7) can be expanded in terms of Gegenbauer polynomials $C_{n}^{3 / 2}$, leading to the following representation for the D-term form factor $D^{q}(t)=\sum_{\substack{n=1 \\ n \text { odd }}}^{\infty} d_{n}^{q}(t)$. Accordingly, the numerical results shown in the next section are for the contribution $d_{1}^{q}(t)$.

\section{Results}

In Fig. 1 we present the dispersive predictions for $d_{1}^{Q}=\sum_{q} d_{1}^{q}(t)$ as function of $t$, with the sum over flavors restricted to up and down quarks. The solid and dashed curves are obtained using as input in Eq. (16) the parametrization of the pion distributions at $Q^{2}=4 \mathrm{GeV}^{2}$ from Refs. [26] and [27], respectively. The different inputs for the pion distributions change the results by an overall normalization factor, without affecting the $t$ dependence. As outlined above, the $Q^{2}$ dependence enters only through the quark momentum fraction of the pion, which changes only by a few percent in the range of $Q^{2}=[1,10] \mathrm{GeV}^{2}$. At $t=0$, we find $d_{1}^{Q}=-1.59$ and $d_{1}^{Q}(0)=-1.92$ for the solid and dashed curve in Fig. 1, respectively. These values compare with the results obtained, at a low normalization scale, in the $\chi \mathrm{QSM}[28], d_{1}^{Q}(0)=-2.35$, in the Skyrme model [29], $d_{1}^{Q}(0)=-4.48$, and in a recent calculation 

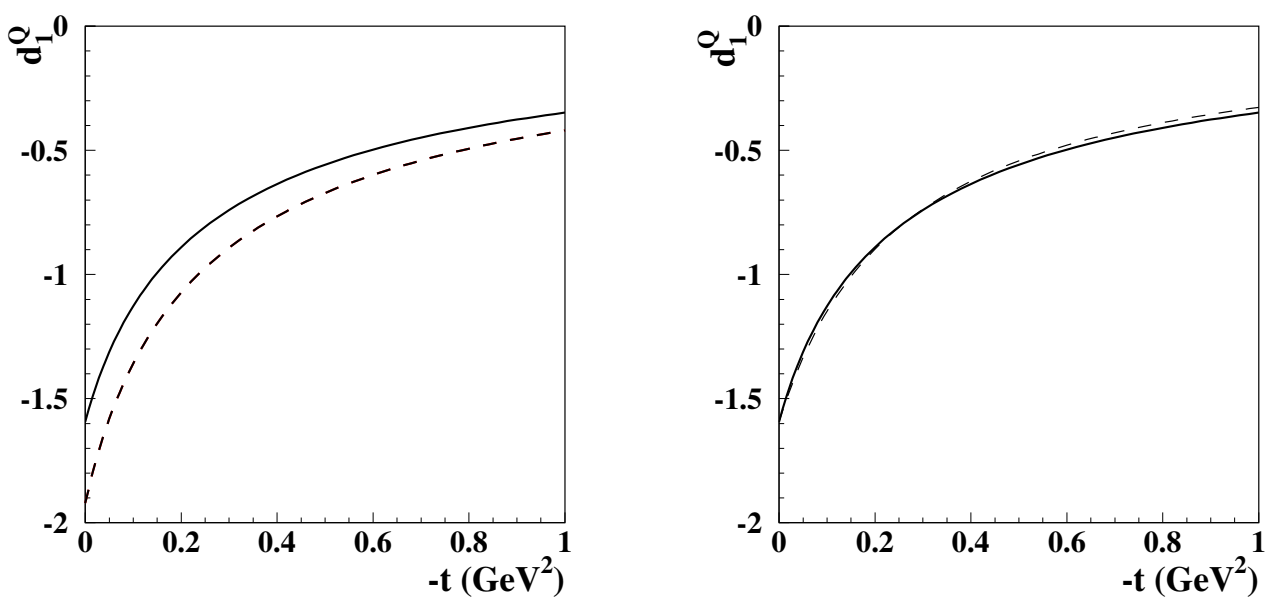

Figure 1. $d_{1}^{Q}$ as function of $t$, at $Q^{2}=4 \mathrm{GeV}^{2}$, obtained with the quark distributions in the pion $q_{\pi}^{f}$ from Ref. [26] (solid curve) and Ref. [27] (dashed curve). Right panel: $t$ dependence of the DR results for $d_{1}^{Q}(t)$ with $q_{\pi}^{f}$ from Ref. [26] (solid curve) in comparison with the function in Eq. (18) (dashed curve).

with effective light-front wave functions from a Regge-improved diquark model [30], $d_{1}^{q}(0)=-2.01$. $d_{1}^{Q}(t)$ is particularly interesting, as it enters in the parametrization of the quark part of the energy momentum tensor of QCD, and provides information on how strong forces are distributed and stabilized in the nucleon [31]. In all theoretical studies so far, as well as in the present dispersive calculation, $d_{1}^{Q}(t)$ at zero-momentum transfer $t=0$ is found to have a negative sign. The negative value of this constant has a deep relation to the spontaneous breaking of the chiral symmetry in QCD [31, 32], and has also an appealing connection with the criterion of stability of the nucleon [28].

In most of phenomenological studies of DVCS, the $t$ dependence of D-term form factor is parametrized by a dipole function [14]. However, the dispersive results favor a different functional form, as shown in the right panel of Fig. 1 where we compare the result for $d_{1}^{Q}$ as function of $t$ with the following parametrization

$$
F_{D}=\frac{d_{1}^{Q}(0)}{\left[1-t /\left(\alpha M_{D}^{2}\right)\right]^{\alpha}}, \text { with } M_{D}=0.487 \mathrm{GeV} \text { and } \alpha=0.841
$$

\section{Conclusions}

We presented a dispersive representation for the quark contribution to the D-term form factor in hard exclusive reactions in terms of unsubtracted $t$-channel dispersion relations. The unitarity relation for the $t$-channel amplitudes is saturated with two-pion intermediate states, taking into account the contribution from $S$ - and $D$-wave intermediate states. The input for the imaginary part of the dispersion relation are the two-pion GDAs, determined through the first- $x$ moment of the flavor-singlet pion PDFs, the $\pi \pi$ phase shifts, and the partial waves for the $\pi \pi \rightarrow N \bar{N}$ amplitudes obtained from dispersion theory by analytical continuation of $\pi N$ scattering amplitudes. We found that the $t$ and 
$Q^{2}$ dependence of the D-term form factor are disjoined. The $t$-dependence is not trivial and does not follow a dipole behavior as normally assumed in phenomenological parametrizations. The value at $t=0$ is also compatible with estimates in chiral-quark soliton model and a Regge-improved diquark model. The $Q^{2}$ dependence enters only in the normalization point at $t=0$, which is proportional to the first $x$-moment of the flavor-singlet pion PDFs.

\section{References}

[1] D. Drechsel, B. Pasquini and M. Vanderhaeghen, Phys. Rept. 378, 99 (2003).

[2] B. Pasquini, M. Gorchtein, D. Drechsel, A. Metz and M. Vanderhaeghen, Eur. Phys. J. A 11, 185 (2001); Phys. Rev. C 62, 052201 (2000).

[3] D. Drechsel, M. Gorchtein, B. Pasquini and M. Vanderhaeghen, Phys. Rev. C 61, 015204 (1999).

[4] B. Pasquini, D. Drechsel and M. Vanderhaeghen, Phys. Rev. C 76, 015203 (2007).

[5] B. Pasquini, D. Drechsel and S. Scherer, Phys. Rev. C 77, 065211 (2008).

[6] I. V. Anikin and O. V. Teryaev, Phys. Rev. D 76, 056007 (2007).

[7] O. V. Teryaev, hep-ph/0510031.

[8] A. V. Radyushkin, Phys. Rev. D 83, 076006 (2011).

[9] K. Kumericki, D. Müller and K. Passek-Kumericki, Nucl. Phys. B 794, 244 (2008).

[10] D. Müller and K. M. Semenov-Tian-Shansky, Phys. Rev. D 92, no. 7, 074025 (2015).

[11] M. Diehl and D. Y. Ivanov, Eur. Phys. J. C 52, 919 (2007).

[12] M. V. Polyakov and M. Vanderhaeghen, arXiv:0803.1271 [hep-ph].

[13] M. V. Polyakov and C. Weiss, Phys. Rev. D 60, 114017 (1999).

[14] M. Guidal, H. Moutarde and M. Vanderhaeghen, Rept. Prog. Phys. 76, 066202 (2013).

[15] B. Pasquini, M. V. Polyakov and M. Vanderhaeghen, Phys. Lett. B 739, 133 (2014).

[16] W. R. Frazer and J. R. Fulco, Phys. Rev. 117, 1603 (1960).

[17] M. Diehl, T. Gousset, B. Pire and O. Teryaev, Phys. Rev. Lett. 81, 1782 (1998).

[18] M. Diehl, T. Gousset and B. Pire, Phys. Rev. D 62, 073014 (2000).

[19] M. V. Polyakov, Nucl. Phys. B 555, 231 (1999).

[20] N. Kivel, L. Mankiewicz and M. V. Polyakov, Phys. Lett. B 467, 263 (1999).

[21] G. Höhler, Pion-Nucleon Scattering, Landolt-Börnstein, Vol. I/9b2, edited by H. Schopper Springer, Berlin, 1983.

[22] B. Lehmann-Dronke, P. V. Pobylitsa, M. V. Polyakov, A. Schäfer and K. Goeke, Phys. Lett. B 475, 147 (2000).

[23] B. Lehmann-Dronke, A. Schäfer, M. V. Polyakov, K. Goeke, Phys. Rev. D 63, 114001 (2001).

[24] N. Warkentin, M. Diehl, D. Y. Ivanov and A. Schafer, Eur. Phys. J. A 32, 273 (2007).

[25] M. V. Polyakov and C. Weiss, Phys. Rev. D 59, 091502 (1999).

[26] J. F. Owens, Phys. Rev. D 30, 943 (1984).

[27] M. Gluck, E. Reya and A. Vogt, Z. Phys. C 53, 651 (1992).

[28] K. Goeke, J. Grabis, J. Ossmann, M. V. Polyakov, P. Schweitzer, A. Silva and D. Urbano, Phys. Rev. D 75, 094021 (2007).

[29] C. Cebulla, K. Goeke, J. Ossmann and P. Schweitzer, Nucl. Phys. A 794, 87 (2007).

[30] D. Müller and D. S. Hwang, arXiv:1407.1655 [hep-ph].

[31] M. V. Polyakov, Phys. Lett. B 555, 57 (2003).

[32] M. V. Polyakov and A. G. Shuvaev, hep-ph/0207153. 